ORIGINAL RESEARCH

S. Kim
L.A. Loevner
H. Quon
A. Kilger
E. Sherman
G. Weinstein
A. Chalian
H. Poptani
EDIOT'S
CHOCES

\section{Prediction of Response to Chemoradiation Therapy in Squamous Cell Carcinomas of the Head and Neck Using Dynamic Contrast- Enhanced MR Imaging}

BACKGROUND AND PURPOSE: Tumor microenvironment, including blood flow and permeability, may provide crucial information regarding response to chemoradiation therapy. Thus, the objective of this study was to investigate the efficacy of pretreatment DCE-MR imaging for prediction of response to chemoradiation therapy in HNSCC.

MATERIALS AND METHODS: DCE-MR imaging studies were performed on 33 patients with newly diagnosed HNSCC before neoadjuvant chemoradiation therapy by using a $1.5 \mathrm{~T}(n=24)$ or a $3 \mathrm{~T}$ ( $n=$ 9) magnet. The data were analyzed by using SSM for estimation of $K^{\text {trans }}, v_{e^{\prime}}$ and $\tau_{i}$. Response to treatment was determined on completion of chemoradiation as $\mathrm{CR}$, with no evidence of disease (clinically or pathologically), or PR, with pathologically proved residual tumor.

RESULTS: The average pretreatment $K^{\text {trans }}$ value of the CR group $\left(0.64 \pm 0.11\right.$ minutes $\left.^{-1}, n=24\right)$ was significantly higher $(P=.001)$ than that of the PR $\left(0.21 \pm 0.05\right.$ minutes $\left.^{-1}, n=9\right)$ group. No significant difference was found in other pharmacokinetic model parameters: $v_{e}$ and $\tau_{i}$, between the 2 groups. Although the PR group had larger metastatic nodal volume than the CR group, it was not significantly different $(P=.276)$.

CONCLUSIONS: These results indicate that pretreatment DCE-MR imaging can be potentially used for prediction of response to chemoradiation therapy of HNSCC.

\begin{abstract}
ABBREVIATIONS: AIF = arterial input function; $\mathrm{CE}=$ contrast-enhanced; chemo $=$ chemotherapy; $\mathrm{CM}=$ cetuximab; Conc. Chemo = concurrent chemotherapy; $\mathrm{CP}=$ cisplatin; $\mathrm{CR}=$ complete response; DCE = dynamic contrast-enhanced; DM = distant metastasis; DT = docetaxol; EGFR = epidermal growth factor receptor; $5 F U=5$-fluorouracil; Gd-DTPA = gadolinium diethylene triamine pentaacetic acid; GKM = general kinetic model; HNSCC = head and neck squamous cell carcinomas; immuno = immunotherapy; Ind. Chemo = induction chemotherapy; $\mathrm{K}^{\text {trans }}=$ transfer constant; $6 \mathrm{MFU}=6$-month follow-up; $\mathrm{N}=$ negative; $\mathrm{NED}=$ no evidence of disease; $\mathrm{P}=$ positive; $\mathrm{PR}=$ partial response; $\mathrm{Rs}=$ treatment response; $\mathrm{Sg}=$ surgery; $\mathrm{Pa}=$ pathology; $\mathrm{SSM}=$ shutter speed model; $\mathrm{T} 1 \mathrm{WI}=\mathrm{T} 1$-weighted imaging; $\mathrm{T} 2 \mathrm{WI}=\mathrm{T} 2$-weighted imaging; $\mathrm{TX}=\mathrm{Taxol} ; v_{e^{\prime}}=$ extravascular extracellular space volume fraction; $\tau_{i \text {, }}=$ intracellular water lifetime; $Y=$ yes
\end{abstract}

$\mathbf{T}$ he efficacy of chemoradiation therapy relies on effective delivery of therapeutic agents and oxygen to the tumor. However, delivery of the drug and oxygen is often impeded by abnormal blood vessels and the presence of tumor cells farther away from functional vasculature. ${ }^{1}$ It has been demonstrated that the rapid onset or reversal of tumor vascular normalization during antiangiogenic therapy can be detected by MR imaging techniques. ${ }^{2}$ Thus, the ability to measure tumor vessels and related tumor microenvironment may provide critical information on the selection of the most effective treatment strategy. ${ }^{1-3}$

Gadolinium-containing paramagnetic molecules have been

Received March 23, 2009; accepted after revision July 1.

From the Departments of Radiology (S.K., L.A.L., A.K., H.P.), Radiation Oncology (H.Q.), Hematology and Oncology (E.S.), and Otolaryngology (G.W., A.C.), University of Pennsylvania, Philadelphia, Pennsylvania; and Center for Biomedical Imaging (S.K.), Department of Radiology, New York University, New York, New York.

This work was supported by National Institutes of Health grant R01-CA102756.

Please address correspondence to Sungheon Kim, PhD, New York University, Department of Radiology, 660 First Ave, 4th Floor, New York, NY 10016; e-mail: Sungheon.Kim@ nyumc.org

Indicates open access to non-subscribers at www.ajnr.org

DOI 10.3174/ajnr.A1817 used as contrast agents to measure blood flow parameters such as perfusion, permeability, or vascular volume in DCE-MR imaging studies. ${ }^{4-6}$ Estimation of tumor microenvironment-related biophysical parameters involves pharmacokinetic modeling of the tumor gadolinium concentration with respect to the plasma compartment. $^{4-6}$ These analyses usually provide estimates of $K^{\text {trans }}$ and $v_{e}$. In addition, DCE-MR imaging data can also be used to measure $\tau_{i}$ by using the SSM, ${ }^{7,8}$ which takes into account the effect of water exchange between intra- and extracellular compartments. These parameters, in isolation or in combination, may play an important role in the prediction and detection of treatment response.

DCE-MR imaging has been used for the diagnosis of cancer $^{9}$ and for monitoring therapeutic response. ${ }^{10}$ In head and neck cancer, heuristic DCE parameters, such as peak enhancement, maximum upslope, time-to-peak enhancement, and washout slope have been used to differentiate cancerous from noncancerous nodes ${ }^{11}$ and for monitoring treatment response. ${ }^{12}$ More recently, pharmacokinetic models have been used to extract parameters related to tumor microcirculation by using DCE-MR imaging in head and neck cancers. ${ }^{13,14}$ However, to our knowledge, there has been no report on the relationship between the pretreatment DCE-MR imaging- 


\begin{tabular}{|c|c|c|c|c|c|c|c|c|c|c|}
\hline No. & Sex & Age (yr) & Primary & Tumor Staging & Ind. Chemo & Conc. Chemo & $\mathrm{Sg}$ & $\mathrm{Pa}$ & Rs & $6 \mathrm{MFU}$ \\
\hline 1 & $M$ & 31 & Larynx & T4aN2bM0 & No & $\mathrm{CP}$ & $\mathrm{N}$ & & $\mathrm{CR}$ & NED \\
\hline 2 & M & 72 & Unknown & TxN2bM0 & $\mathrm{CM}$ & No & $\mathrm{N}$ & & $\mathrm{CR}$ & NED \\
\hline 3 & $\mathrm{~F}$ & 68 & Tongue & T4N2bM0 & No & $\mathrm{CP}$ & $\mathrm{N}$ & & $\mathrm{CR}$ & $\mathrm{DM}$ \\
\hline 4 & $M$ & 68 & Tonsil & T2N2bM0 & No & $\mathrm{CP}$ & $\mathrm{N}$ & & $\mathrm{CR}$ & NED \\
\hline 5 & M & 70 & Tonsil & T4N1aM0 & No & $\mathrm{CP}$ & $\mathrm{N}$ & & $\mathrm{CR}$ & NED \\
\hline 6 & M & 67 & Tongue & T4aN2cM0 & No & $\mathrm{CP}$ & $\mathrm{N}$ & & $\mathrm{CR}$ & NED \\
\hline 7 & $\mathrm{~F}$ & 79 & Unknown & T4aN2bM0 & $\mathrm{CM}$ & $\mathrm{CM}, \mathrm{CP}$ & $\mathrm{N}$ & & $\mathrm{CR}$ & NED \\
\hline 8 & M & 69 & Larynx & T3N2cMX & CP, DT, 5FU & $\mathrm{CM}$ & $\mathrm{N}$ & & $\mathrm{CR}$ & NED \\
\hline 9 & M & 72 & Tongue & T4N2cM0 & CP, DT, 5FU & $\mathrm{CM}$ & $\mathrm{N}$ & & $\mathrm{CR}$ & NED \\
\hline 10 & M & 67 & Unknown & TON2bMO & $\mathrm{CP}, \mathrm{DT}, 5 \mathrm{FU}$ & $\mathrm{CM}$ & $\mathrm{N}$ & & $\mathrm{CR}$ & NED \\
\hline 11 & $\mathrm{M}$ & 72 & Tongue & T2N2bMO & No & $\mathrm{CM}$ & $\mathrm{N}$ & & $\mathrm{CR}$ & NED \\
\hline 12 & $\mathrm{M}$ & 75 & Tongue & T4N2cM0 & TX & TX, CP & Y & $N$ & $\mathrm{CR}$ & NED \\
\hline 13 & M & 42 & Tonsil & T2N2bM0 & No & $\mathrm{CP}$ & Y & $\mathrm{N}$ & $\mathrm{CR}$ & NED \\
\hline 14 & $\mathrm{~F}$ & 53 & Tonsil & TxN2bM0 & No & $\mathrm{CP}$ & Y & $N$ & CR & NED \\
\hline 15 & $\mathrm{M}$ & 50 & Tongue & T2N2aMO & CP, DT, 5FU & $\mathrm{CP}$ & Y & $N$ & $\mathrm{CR}$ & NED \\
\hline 16 & M & 59 & Tongue & T3N2cM0 & $\mathrm{CP}, \mathrm{DT}, 5 \mathrm{FU}$ & $\mathrm{CP}$ & Y & $\mathrm{N}$ & $\mathrm{CR}$ & NED \\
\hline 17 & $\mathrm{M}$ & 51 & Tongue & TxN2bMO & $\mathrm{CP}, \mathrm{DT}, 5 \mathrm{FU}$ & $\mathrm{CP}$ & Y & $\mathrm{N}$ & $\mathrm{CR}$ & $\mathrm{DM}$ \\
\hline 18 & $\mathrm{M}$ & 55 & Tonsil & T3N2bM0 & $\mathrm{CP}, \mathrm{TX}$ & $\mathrm{CP}$ & Y & $N$ & $\mathrm{CR}$ & NED \\
\hline 19 & $\mathrm{~F}$ & 62 & Tonsil & T2N2cM0 & No & $\mathrm{CP}$ & Y & $\mathrm{N}$ & $\mathrm{CR}$ & NED \\
\hline 20 & $\mathrm{M}$ & 65 & Unknown & TxN2bM0 & No & $\mathrm{CP}$ & Y & $\mathrm{N}$ & $\mathrm{CR}$ & NED \\
\hline 21 & M & 52 & Tongue & T1N2abM0 & CP, DT, 5FU & $\mathrm{CP}$ & Y & $N$ & $\mathrm{CR}$ & NED \\
\hline 22 & M & 59 & Tongue & T4N2bM0 & No & $\mathrm{CP}$ & Y & $N$ & $\mathrm{CR}$ & NED \\
\hline 23 & $\mathrm{M}$ & 49 & Tonsil & T3N2aM0 & No & $\mathrm{CM}$ & Y & $\mathrm{N}$ & $\mathrm{CR}$ & NED \\
\hline 24 & M & 61 & Tongue & T3N2aM0 & $\mathrm{CP}$ & $\mathrm{CM}, \mathrm{CP}$ & Y & $\mathrm{N}$ & $\mathrm{CR}$ & NED \\
\hline 25 & $M$ & 51 & Epiglottis & T2N2cM0 & TX & $\mathrm{CP}$ & Y & $P$ & PR & $\mathrm{DM}$ \\
\hline 26 & $\mathrm{M}$ & 76 & Tonsil & TxN2bM0 & TX & $\mathrm{CP}, \mathrm{TX}$ & Y & $P$ & PR & $\mathrm{DM}$ \\
\hline 27 & M & 77 & Larynx & T2N2bMO & No & $\mathrm{CP}, 5 \mathrm{FU}$ & Y & $P$ & $\mathrm{PR}$ & NED \\
\hline 28 & M & 56 & Larynx & T2N3M0 & No & $\mathrm{CP}$ & Y & $\mathrm{P}$ & PR & $\mathrm{DM}$ \\
\hline 29 & M & 64 & Unknown & TxN3M0 & $\mathrm{CP}, \mathrm{DT}, 5 \mathrm{FU}$ & $\mathrm{CP}$ & Y & $P$ & PR & $\mathrm{DM}$ \\
\hline 30 & $\mathrm{M}$ & 60 & Tonsil & T4N1M0 & No & $\mathrm{CP}$ & Y & $P$ & $\mathrm{PR}$ & NED \\
\hline 31 & $M$ & 63 & Larynx & T2N2bM0 & $\mathrm{CP}$ & $\mathrm{CP}$ & Y & $P$ & PR & $\mathrm{DM}$ \\
\hline 32 & $\mathrm{M}$ & 45 & Tongue & T2N2aM0 & No & $\mathrm{CM}, \mathrm{CP}$ & Y & $P$ & PR & $\mathrm{DM}$ \\
\hline 33 & $\mathrm{~F}$ & 48 & Tonsil & T4bN2bM0 & CP, DT, 5FU & $\mathrm{CM}, \mathrm{CP}$ & $\mathrm{N}^{\mathrm{a}}$ & & $\mathrm{PR}$ & Relapse \\
\hline
\end{tabular}

${ }^{a}$ Nodes were not removed because additional therapy was given due to disease progression.

derived pharmacokinetic parameters of the metastatic node and treatment outcome in patients with head and neck cancer. The availability of such prognostic imaging markers could significantly contribute to the selection of the most effective treatment strategy for a given patient. For example, in HNSCC, organ-preserving radiation therapy with concurrent chemotherapy has become an accepted standard treatment option for patients with metastatic cervical nodes. ${ }^{15}$ However, it remains unclear if all patients with locally advanced HNSCC benefit from this treatment paradigm. ${ }^{16,17}$ Development of imaging biomarkers can potentially help in "triaging" the nonresponsive tumors to alternative strategies, such as resection or tumor-targeted therapies, including EGFR-based immunotherapies. Thus, the present study was conducted to evaluate the utility of DCE-MR imaging-based pharmacokinetic parameters as potential biomarkers for predicting response to chemoradiation therapy in HNSCC.

\section{Materials and Methods}

\section{Patient Population and Treatment}

The institutional review board approved this study, and written informed consent was obtained from all subjects before MR imaging studies. The study involved 33 patients ( 5 women and 28 men, $60.8 \pm$ 10.8 years of age) who were newly diagnosed with HNSCC with no prior treatment and were referred for preoperative chemoradiation therapy. All patients were assessed by a radiation oncologist and a neuroradiologist for the presence of metastatic nodes on the basis of clinical reports and radiologic examination. The largest node was identified by the neuroradiologist as the target for the study. Treatment included accelerated radiation therapy with $220 \mathrm{cGy}$ per fraction for a total dose of $7040 \mathrm{cGy}$ to the gross tumor volume in 32 fractions during 44 days, with induction and/or concurrent chemotherapy as shown in Table 1. All patients underwent MR imaging studies before initiation of treatment.

The current study was performed to assess the prognostic value of pretreatment MR imaging parameters in predicting treatment response of the metastatic node to neoadjuvant chemoradiation therapy. Disease status at the end of chemoradiation therapy was used as the clinical end point because additional therapies, such as surgery or chemotherapy, performed in patients with any evidence of residual disease after the end of neoadjuvant chemoradiation therapy can act as confounders in assessing the role of neoadjuvant chemoradiation therapy alone. The patients were retrospectively categorized into 2 groups: CR, with no evidence of disease $(n=24)$ or PR, with evidence of residual disease $(n=9)$. CR included patients who showed no viable tumor on pathology from the surgically removed nodes $(n=13)$ and those who did not undergo surgery due to no evidence of disease $(n=11)$. All PR cases were confirmed by pathology for the presence of a viable tumor from the surgically removed nodes, except 1 patient whose nodes were not removed and for whom additional therapy was given as the disease progressed. 

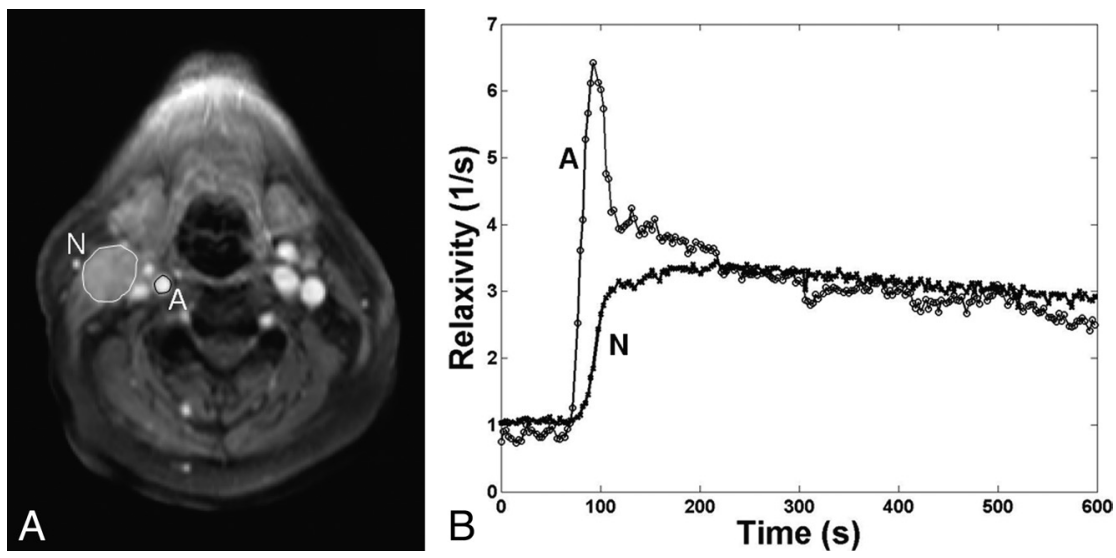

Fig 1. A, Representative regions of interest shown on a T1WI of the neck acquired 10 minutes after injection of the contrast agent. Regions of interest are drawn on a carotid artery (A) and a metastatic node (N). B. Mean time courses of estimated relaxation rates from the 2 regions of interest in $A$ are plotted against time. Circles and crosses represent actual measurement data points from the selected artery and the node, with the lines connecting the points. Note the region of interest used for the node is only to demonstrate the typical enhancement pattern and temporal resolution of the data. The actual pharmacokinetic analysis was performed for each voxel.

Posttherapy surgery was performed an average of $65 \pm 23$ days after the completion of chemoradiation therapy in all patients. Of the 9 PR patients, $78 \%$ presented with metastasis within 6 months, even after complete dissection of the remaining nodes. In contrast, only 2 patients from the CR group (8\%) developed distant metastasis within the first 6 months after completion of therapy, while the remaining CR patients had no evidence of disease at 6-month follow-up assessment. This observation indicates that the treatment response, assessed at the end of neoadjuvant chemoradiation therapy for individual nodes, strongly correlated with the 6-month follow-up.

\section{Data Acquisition}

The MR imaging study was performed by using a 1.5T Sonata scanner $(n=24)$ or a 3 T Trio scanner $(n=9)$ (Siemens Medical Systems, Iselin, New Jersey). A neck array coil or a neurovascular coil was used for $1.5 \mathrm{~T}$ or $3 \mathrm{~T}$ scanners, respectively. Axial T2W and T1W was acquired by using a spin-echo sequence (TR/TE $=4$ seconds $/ 120 \mathrm{~ms}$ for $\mathrm{T} 2 \mathrm{~W}$, and TR/TE $=600 \mathrm{~ms} / 10 \mathrm{~ms}$ for T1W). Eight axial sections with $\mathrm{FOV}=26 \mathrm{~cm}$ and section thickness $=5 \mathrm{~mm}$ were selected to cover the metastatic cervical lymph node for T1, T2, and DCE-MR imaging. Measurement of T2 was performed by acquiring a series of T2W images by using a spin-echo sequence with 4 different TEs: 13, 53, 80, and $110 \mathrm{~ms}$ ( $\mathrm{TR}=2$ seconds). Before the injection of the contrast agent, measurement of T1 was performed by acquiring a series of T1W images by using an inversion-recovery prepared turbo fast low-angle shot 3D sequence with 5 different inversion times: 0.06 , $0.2,0.4,0.8$, and 1.6 seconds. DCE-MR imaging was performed by using a fast $3 \mathrm{D}$ spoiled gradient-echo sequence, which was modified to acquire 8 angle-interleaved subaperture images from the full-echo radial data. ${ }^{18}$ The imaging parameters were the following: 256 readout points/view, 256 views (32 views/subaperture, 8 subapertures), $\mathrm{FOV}=26 \mathrm{~cm}$, section thickness $=5 \mathrm{~mm}, 8$ axial sections, flip angle $=$ $20^{\circ}$, receiver bandwidth $=510 \mathrm{~Hz} /$ pixel, $\mathrm{TR}=5.0 \mathrm{~ms}$, and $\mathrm{TE}=$ $4.2 \mathrm{~ms}$. Fat saturation was applied once every 8 excitations. Spatial saturation was applied once every 32 excitations to minimize the flow effect while minimizing the scanning time.

The scanning time of full-resolution data was approximately 20 seconds with fat and spatial saturations. This data-acquisition scheme resulted in a temporal resolution of 2.5 seconds for each subaperture image with full spatial resolution of $256 \times 256$ by using a previously reported dynamic $k$-space-weighted image reconstruction contrast algorithm. ${ }^{18}$ Baseline preinjection images were acquired for 1 minute. A single dose of Gd-DTPA (Omniscan; GE Healthcare, Milwaukee, Wisconsin) with a concentration of $0.1 \mathrm{mmol} / \mathrm{L} / \mathrm{kg}$ body weight was injected with a power injector (Medrad, Indianola, Pennsylvania) at the rate of $1 \mathrm{~mL} / \mathrm{s}$ into an antecubital vein, followed by a saline flush during which scanning was continued for another 9 minutes.

\section{Data Analysis}

Because images from the head and neck region are subject to voluntary and involuntary motion such as swallowing and breathing, all images were coregistered by using a 2-step nonrigid image registration technique before data analysis. ${ }^{14,19}$ Regions of interest for metastatic nodal masses were drawn by a neuroradiologist (L.A.L.) on the basis of T1WI, T2WI, and T1WI-gadolinium images, which were used for measurement of tumor volume and the analysis of parametric maps. Pharmacokinetic analysis was performed for each voxel in the selected region of interest. The spoiled gradient-echo signal intensity was converted to a function of the apparent longitudinal relaxation rate based on the TR and flip angle. ${ }^{20}$ These relaxation-rate curves of individual voxels in metastatic cervical lymph nodes were converted to the contrast agent concentration curves and were then fitted to a 2-compartment pharmacokinetic model.

The SSM accounts for the effect of transcytolemmal water exchange, which makes the relationship between the contrast agent concentration and relaxation rates deviate from linearity. ${ }^{7,14}$ It has been reported that the SSM adequately fits the dynamic contrast-enhanced MR imaging data from HNSCC. ${ }^{14}$ In this study, we used the fast exchange regime-allowed model under the assumption that contrast agent concentrations are within the range commonly used for human studies $(<5 \mathrm{mmol} / \mathrm{L}){ }^{7,14}$ The contribution from the vascular volume was assumed to be negligible in tumor tissues with relatively high $K^{\text {trans }},{ }^{21}$ because the water exchange between the vascular and interstitial compartments becomes faster with increased vascular permeability. An additional parameter estimated in SSM is the mean $\tau_{i}$.

The parameters that were held constant during the analysis were hematocrit $(0.45)$, tissue water volume fraction $(0.8)$, extravascular extracellular space, blood contrast agent relaxivity $(4.1$ and $4.3 \mathrm{mmol} /$ $\mathrm{L}^{-1} \mathrm{~s}^{-1}$ for 1.5 and $3 \mathrm{~T}$, respectively), and blood T1 (1.2 and $1.8 \mathrm{sec}-$ onds for 1.5 and $3 \mathrm{~T}$, respectively) based on the literature data. ${ }^{22,23}$ Thus, the remaining parameters to be estimated were $K^{\text {trans }}, v_{e}$ and $\tau_{i}$. The AIF was semiautomatically obtained from a region of interest 

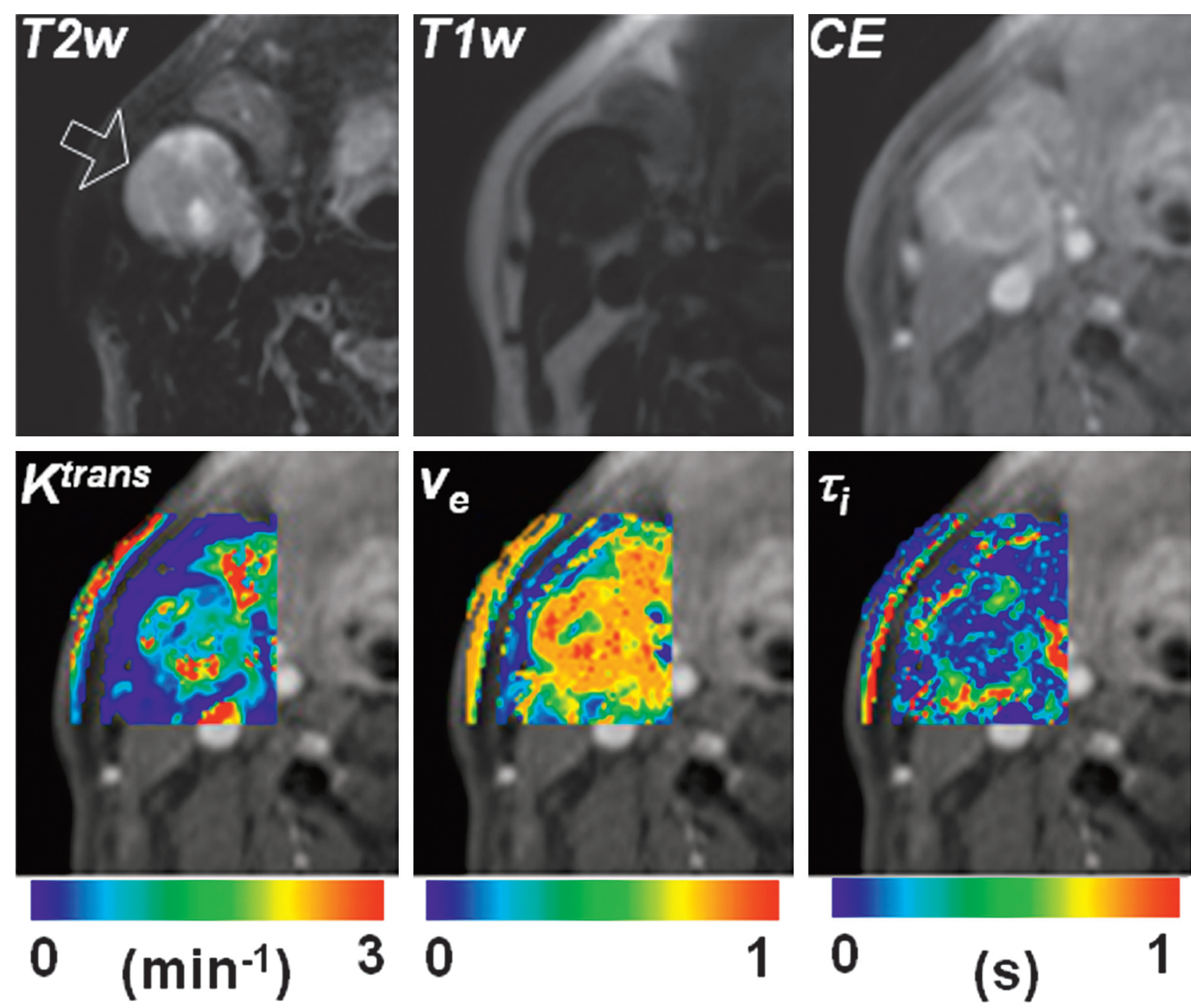

Fig 2. Representative parametric maps of a CR patient who showed no viable tumor on pathology from the surgically removed nodes. The arrow on the T2WI image indicates the tumor node studied. The T2W, T1W, and CE images in the top row are windowed to have similar image contrast. The SSM parameter maps are shown by using overlaid color images on an area around the metastatic node indicated by the arrow in the T2WI. The background image of the color maps is a T1W CE image.

drawn on one of the carotid arteries located in close proximity to the tumor according to the method proposed by Rijpkema et al. ${ }^{13}$ Using the AIF, the SSM parameters were estimated by minimizing the average weighted residual sum of squares. ${ }^{14}$ Parameter estimation was performed by using a Simplex algorithm ${ }^{24}$ provided in IDL (RSI, Boulder, Colorado). The estimated pharmacokinetic model parameters from 1.5 and $3 \mathrm{~T}$ were combined because differences in the magnetic field were accounted for by using the published relaxivity values at the 2 field strengths and individually measured $\mathrm{T} 1$ relaxation times. The differences between the groups were assessed by using a 2-tailed $t$ test with unequal variance.

\section{Results}

A representative example of contrast-enhancement curves from a carotid artery and a metastatic node is shown in Fig 1 , which demonstrates that the temporal resolution of image acquisition was adequate for measuring the fast-rising edge of the enhancement curves from the tumor and the narrow peak of the AIF. Although the example enhancement curves are shown from regions of interest, the phar- macokinetic analysis was performed for each pixel. Representative examples of pharmacokinetic parameter maps from a CR patient (a 59-year-old man with a primary at the base of the tongue) are shown in Fig 2. This patient received surgical dissection of the residual mass after chemoradiation but did not have any viable tumor on pathologic assessment. The parameter maps demonstrate tumor heterogeneity within the small node studied.

Figure 3 shows scatterplots for the pretreatment volume and SSM parameters of all patients. Both CR and PR groups had tumor similar volume, $v_{e}$ and $\tau_{i}$, at the time of presentation; however, $K^{\text {trans }}$ values were generally higher in CR. A higher pretreatment $K^{\text {trans }}$ value appears to be indicative of a good treatment response. Table 2 shows the mean and standard error of the parameters shown in Fig 3. The $K^{\text {trans }}$ values of the CR group were significantly higher than those of the PR $(P=.001)$ group. No significant difference was observed in the tumor volume, $v_{e}$, and $\tau_{i}$ between the 2 groups $(P>.05)$.

Figure 4 shows a comparison of pretreatment MR imaging 

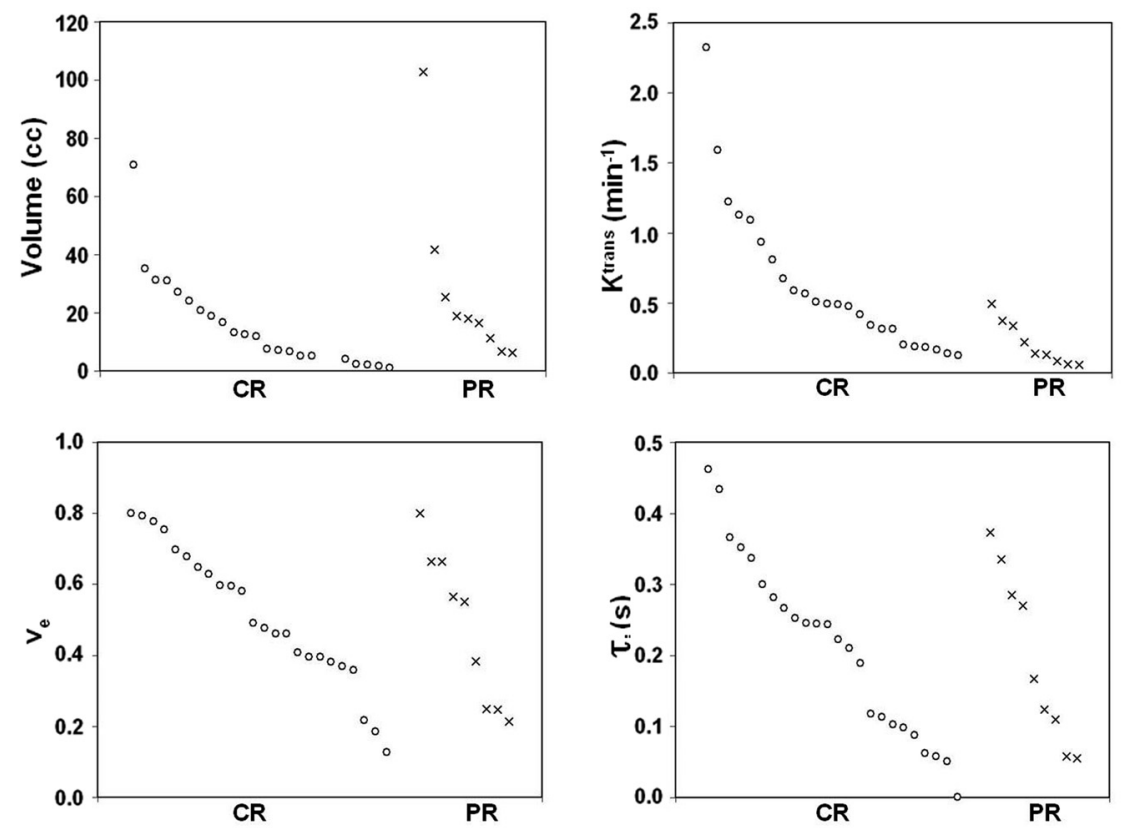

Fig 3. Scatterplots of volume and pharmacokinetic model parameters of 2 patient groups: $C R(n=24)$ and PR $(n=9)$. Individual parameters within each patient group are plotted in their own descending orders.

\begin{tabular}{|c|c|c|c|c|c|}
\hline Group & No. of Patients & Volume (mL) & $K^{\text {trans }}\left(\min ^{-1}\right)$ & $v_{e}$ & $\tau_{l}(\mathrm{~s})$ \\
\hline $\mathrm{CR}$ & 24 & $15.3 \pm 3.2$ & $0.64 \pm 0.11$ & $0.51 \pm 0.04$ & $0.21 \pm 0.0$ \\
\hline PR & 9 & $27.5 \pm 10.1$ & $0.21 \pm 0.05$ & $0.48 \pm 0.07$ & $0.20 \pm 0.0$ \\
\hline$P$ value $^{b}$ & & .276 & .001 & & \\
\hline
\end{tabular}

The reported values are mean and standard error.

b The $P$ values were computed using a 2-tailed $t$ test with unequal variance.

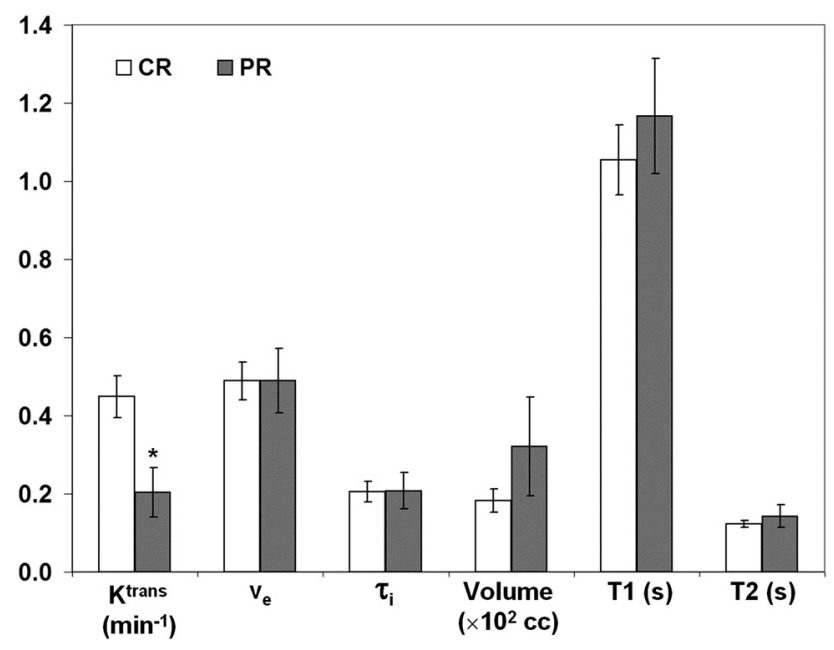

Fig 4. Comparison of patients scanned at 1.5T. There are 17 and 7 patients for the CR and PR groups, respectively. The bars represent the mean values and the error bars represent the standard errors. The asterisk represents a significant $(P<.05$, 2-tailed $t$ test with unequal variance) difference from the CR mean value.

parameters of the patients who were scanned by using a $1.5 \mathrm{~T}$ scanner $(n=24)$. Similar to what was observed with all the patients by using both scanners, the CR group had significantly higher $K^{\text {trans }}$ values than the $\operatorname{PR}(P=.011)$ group. The mean T1 and T2 values of the PR group were higher than those of the CR group by $11 \%$ and $15 \%$, respectively. However, these were not significantly different $(P>.05)$. A similar trend was also observed with the patients who underwent MR imaging studies by using a $3 \mathrm{~T}$ scanner $(n=9)$. However, the number of patients for each treatment response group $(n=7$ and 2 for $\mathrm{CR}$ and $\mathrm{PR}$, respectively) was too small to conduct a statistical analysis. These results indicate that the results were likely independent of the magnetic field.

We also tested the value of DCE imaging in differentiating the patients receiving immunotherapy with cetuximab, a monoclonal antibody for EGFR, and the ones who underwent conventional chemotherapy (cisplatin, docetaxol, paclitaxel [Taxol], or 5FU). Figure 5 shows a comparison of the $K^{\text {trans }}$ values of the patients who received chemotherapy ( $n=17$, and 7 for CR and PR, respectively) and the patients whose systemic therapy included cetuximab ( $n=7$ and 2 for CR and $\mathrm{PR}$, respectively). For the patients undergoing conventional chemotherapy, the CR group had significantly higher $K^{\text {trans }}$ $(P=.021)$ values than the PR groups. The number of patients with cetuximab was too small to conduct a statistical analysis. However, these early results demonstrate that the patients with cetuximab had a trend similar to that observed in the patients with conventional chemotherapy, indicating that pretreatment $K^{\text {trans }}$ values can likely predict response regardless of the type of chemotherapy.

\section{Discussion}

In this study, we investigated the efficacy of pharmacokinetic parameters derived from pretreatment DCE-MR imaging data for prediction of treatment response to chemoradiation ther- 


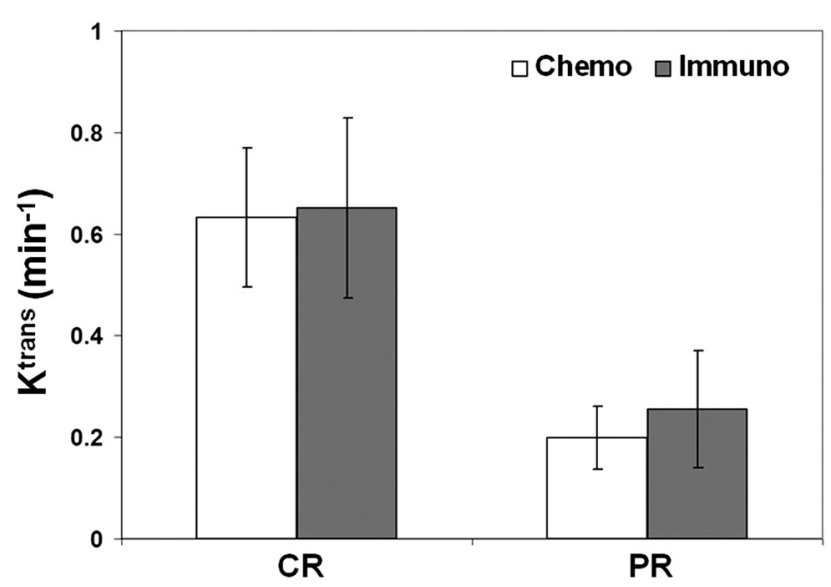

Fig 5. Comparison of pretreatment $K^{\text {trans }}$ values of the patients who received conventional chemotherapy ( $n=17$ and 7 for CR and PR, respectively) and those who received immunotherapy with an EGFR-targeting drug, cetuximab ( $n=7$ and 2 for CR and PR, respectively). The vertical bars represent the mean values, and the error bars represent the SDs.

apy in patients with HNSCC. The patients who responded favorably to chemoradiation therapy had significantly higher pretreatment $K^{\text {trans }}$ values than the patients with partial or no response. No significant difference was found in other parameters, including volume of the metastatic node and the T1 and T2 relaxation values. These results suggest that the pharmacokinetic parameters have a potential as prognostic biomarkers for predicting the therapeutic response in HNSCC.

Given the importance of tumor oxygenation and tumor microvessel attenuation in predicting clinical response to radiation, ${ }^{25}$ DCE-MR imaging can potentially play a critical role in predicting responses to chemo- and/or radiation therapy. The role of pretreatment DCE-MR imaging in predicting therapeutic response in colorectal and cervical cancers has been reported previously. ${ }^{26-28}$ From a DCE-MR imaging study of patients with uterine cervical cancer undergoing surgery, Yamashita et $\mathrm{al}^{26}$ reported that areas of high $K^{\text {trans }}$ were predominantly composed of cancer cell fascicles, whereas areas of low $K^{\text {trans }}$ were composed of fibrous tissue with scattered cancer cells. Furthermore, radiation therapy was more effective in tumors with high $K^{\text {trans }}$ than in tumors exhibiting low $K^{\text {trans }}$ values. Similarly, George et $\mathrm{al}^{27}$ reported a significant correlation between serum vascular endothelial growth factor and $K^{\text {trans }}$ before treatment in patients with colorectal cancer treated by chemoradiation therapy. This study also reported significantly higher pretreatment $K^{\text {trans }}$ in responders than in nonresponders $(P=.03)$, along with a significant reduction in $K^{\text {trans }}$ by the end of the treatment in responders. ${ }^{27}$

In another study, Loncaster et $\mathrm{al}^{28}$ reported that patients with cervical cancer with high pretreatment contrast enhancement had significantly improved disease-free survival $(P=$ $.024)$ after radiation therapy. These reports are in good agreement with the findings of the present study. Most interesting though, a recent DCE-MR imaging study in patients with colorectal liver metastases undergoing 5FU treatment reported that $K^{\text {trans }}$ measured before treatment did not predict treatment response, suggesting that the increased drug delivery due to better perfusion may not be the only factor for successful treatment. ${ }^{29}$ Because the mechanism of action of chemotherapeutic drugs varies significantly depending on the tumor type and the tissue, further investigation is necessary to assess the value of $K^{\text {trans }}$ in predicting treatment response to different therapeutic strategies in various tumor types.

Recently, Cao et al (2008) $)^{30}$ used early changes in blood volume and blood flow between the baseline measurements and 2 weeks into chemoradiation therapy to predict treatment response and reported significant changes in blood volume of the primary tumor as a potential marker for assessing response. Pretreatment deconvolution-based CT perfusion studies of the primary tumor also demonstrated higher blood flow in tumors exhibiting significant treatment response. ${ }^{31}$ The authors of this study had earlier reported a correlation between high pretreatment blood volume and positive tumor response in patients with oropharyngeal carcinomas treated with induction chemotherapy. ${ }^{32}$ Hermans et $\mathrm{al}^{33}$ also reported a correlation between low pretreatment CT perfusion and the increased risk of local recurrence following definitive radiation therapy without induction chemotherapy in patients with HNSCC. Our results are in agreement with these reports because we observed significantly higher pretreatment $K^{\text {trans }}$ values (by using DCE-MR imaging) in CR than in PR. The advantage of MR imaging over CT is superior soft-tissue contrast for anatomic delineation of the tumor while avoiding additional radiation exposure from CT scanning.

DCE-MR imaging is a minimally invasive technique, which provides an opportunity to probe the tumor microenvironment, such as perfusion, permeability of blood vessels, and volume of the extracellular space. ${ }^{4-8,34}$ Different pharmacokinetic models have been suggested to look at interstitial fluid pressure, ${ }^{35}$ contrast agent diffusivity, ${ }^{36}$ and $\tau_{i}{ }^{7,14}$ in addition to $K^{\text {trans }}$ and $v_{e}$. In the present study, we used the SSM, which suggests that the water exchange rate between interstitial and intracellular compartments cannot be ignored with the usual clinical dosage of Gd-DTPA. However, we did ignore the water exchange between the vascular and interstitium compartments because we assumed that the vascular component is negligible in our model. Li et $\mathrm{al}^{21}$ also showed that when $K^{\text {trans }}$ was higher than $0.1 / \mathrm{min}$ and the vascular volume was around 5\%, the SSM was adequate in modeling the data. Because the $K^{\text {trans }}$ values were typically higher, we believe that the assumption of a negligible vascular volume applies to a majority of the cases. For the case with low $K^{\text {trans }}(<0.1 / \mathrm{min})$ but high blood volume $(>5 \%)$, a more complex model, such as the 3-site exchange model, could be used. However, such a scenario is unlikely to be present in tumors. Future studies comparing the SSM with a 3-site exchange model would be necessary to resolve these issues.

Previously, we compared the $K^{\text {trans }}$ values from the GKM and the SSM in HNSCC. ${ }^{14}$ We found that the $K^{\text {trans }}$ values of the SSM were higher than those of the GKM, mainly because the SSM can adequately represent the rapid increase of contrast agent concentration during the wash-in phase compared with the GKM. A similar finding was also reported by other investigators. ${ }^{8,37}$ Thus, higher $K^{\text {trans }}$ values are more consistently observed from SSM than from other models similar to GKM.

We also observed that some regions exhibited very large $v_{e}$. This could be due to the presence of necrosis or other factors that were important but ignored in the pharmacokinetic modeling. Unrealistically high $\mathrm{v}_{\mathrm{e}}$ values have been reported not 
only for the SSM but also for the GKM. ${ }^{36}$ It is possible that the relatively simple models, such as the SSM and Tofts (GKM) cannot adequately represent the complex tissue environment. Pellerin et $\mathrm{al}^{36}$ investigated the effect of diffusion, which is usually ignored in DCE-MR imaging analysis, and found that inclusion of water diffusion reduces the overestimation of $v_{e}$ and $K^{\text {trans }}$. Although useful, these methods require high computational power and may not be suitable for clinical application. Further development of physiologic modeling methods with histologic validation would be necessary to address this issue more systematically.

\section{Conclusions}

At present, there are no noninvasive markers that can reliably predict outcome in cancer therapy. Development of physiologically sensitive MR imaging methods that allow early prediction of response will assist in selection of the optimal therapeutic strategy for HNSCC, including the most effective combination of induction chemotherapy, concurrent chemoradiation therapy, and surgery. The availability of such biologic markers will help in determining those patients who may respond well to a certain type of therapeutic regimen. The results of our current study suggest that the pharmacokinetic parameters, particularly $K^{\text {trans }}$, have a potential to be used as noninvasive biomarkers for predicting therapeutic response in HNSCC. With the development of organ-preserving surgical techniques, the need to identify radiosensitive HNSCC is even more critical to better triage patients with nonresectable HNSCC. These noninvasive markers may play a critical role in patient management and reduce the cost of ineffective therapies for nonresponders, providing them with more effective and viable alternatives.

\section{References}

1. Jain RK. Normalization of tumor vasculature: an emerging concept in antiangiogenic therapy. Science 2005;307:58-62

2. Batchelor TT, Sorensen AG, di Tomaso E, et al. AZD2171, a pan-VEGF receptor tyrosine kinase inhibitor, normalizes tumor vasculature and alleviates edema in glioblastoma patients. Cancer Cell 2007;11:83-95

3. Ludwig JA, Weinstein JN. Biomarkers in cancer staging, prognosis and treatment selection. Nat Rev Cancer 2005;5:845-56

4. Tofts PS, Kermode AG. Measurement of the blood-brain barrier permeability and leakage space using dynamic MR imaging. 1. Fundamental concepts. Magn Reson Med 1991;17:357-67

5. Tofts PS. Modeling tracer kinetics in dynamic Gd-DTPA MR imaging. J Magn Reson Imaging 1997;7:91-101

6. Brix G, Semmler W, Port R, et al. Pharmacokinetic parameters in CNS GdDTPA enhanced MR imaging. J Comput Assist Tomogr 1991;15:621-28

7. Landis CS, Li X, Telang FW, et al. Equilibrium transcytolemmal water-exchange kinetics in skeletal muscle in vivo. Magn Reson Med 1999;42:467-78

8. Yankeelov TE, Rooney WD, Li X, et al. Variation of the relaxographic "shutterspeed" for transcytolemmal water exchange affects the CR bolus-tracking curve shape. Magn Reson Med 2003;50:1151-69

9. Cha S. Update on brain tumor imaging: from anatomy to physiology. AJNR Am J Neuroradiol 2006;27:475-87

10. O'Connor JP, Jackson A, Parker GJ, et al. DCE-MRI biomarkers in the clinical evaluation of antiangiogenic and vascular disrupting agents. $\mathrm{Br} \mathrm{J}$ Cancer 2007;96:189-95

11. Noworolski SM, Fischbein NJ, Kaplan MJ, et al. Challenges in dynamic contrast-enhanced MRI imaging of cervical lymph nodes to detect metastatic disease. J Magn Reson Imaging 2003;17:455-62

12. Hoskin PJ, Saunders MI, Goodchild K, et al. Dynamic contrast enhanced magnetic resonance scanning as a predictor of response to accelerated radiotherapy for advanced head and neck cancer. Br J Radiol 1999;72:1093-98

13. Rijpkema M, Kaanders JH, Joosten FB, et al. Method for quantitative mapping of dynamic MRI contrast agent uptake in human tumors. J Magn Reson Imaging 2001;14:457-63

14. Kim S, Quon H, Loevner LA, et al. Transcytolemmal water exchange in pharmacokinetic analysis of dynamic contrast-enhanced MRI data in squamous cell carcinoma of the head and neck. J Magn Reson Imaging 2007;26:1607-17

15. American Cancer Society. Cancer Facts \& Figures 2008. Available at: http://www. cancer.org/docroot/stt/content/stt_1x_cancer_facts_and_figures_2008.asp. Accessed May 28, 2008

16. Zorat PL, Paccagnella A, Cavaniglia G, et al. Randomized phase III trial of neoadjuvant chemotherapy in head and neck cancer: 10-year follow-up. J Natl Cancer Inst 2004;96:1714-17

17. Bonner JA, Harari PM, Giralt J, et al. Radiotherapy plus cetuximab for squamous-cell carcinoma of the head and neck. New Engl J Med 2006;354:567-78

18. Song HK, Dougherty L. Dynamic MRI with projection reconstruction and KWIC processing for simultaneous high spatial and temporal resolution. Magn Reson Med 2004;52:815-24

19. Kim S, Dougherty L, Rosen MA, et al. Automatic correction of in-plane bulk motion artifacts in self-navigated radial MRI. Magn Reson Imaging 2008;26:367-78

20. Tofts PS, Berkowitz B, Schnall MD. Quantitative analysis of dynamic GdDTPA enhancement in breast tumors using a permeability model. Magn Reson Med 1995;33:564-68

21. Li X, Rooney WD, Springer CS Jr. A unified magnetic resonance imaging pharmacokinetic theory: intravascular and extracellular contrast reagents. Magn Reson Med 2005;54:1351-59

22. Yankeelov TE, Rooney WD, Huang W, et al. Evidence for shutter-speed variation in CR bolus-tracking studies of human pathology. NMR Biomed 2005; 18:173-85

23. Cashen TA, Carr JC, Shin W, et al. Intracranial time-resolved contrast-enhanced MR angiography at 3T. AJNR Am J Neuroradiol 2006;27:822-29

24. Nelder JA, Mead R. A simplex method for function minimization. Comput J 1965;7:308-13

25. Kamijo T, Yokose T, Hasebe T, et al. Potential role of microvessel density in predicting radiosensitivity of $\mathrm{T} 1$ and $\mathrm{T} 2$ stage laryngeal squamous cell carcinoma treated with radiotherapy. Clin Cancer Res 2000;6:3159-65

26. Yamashita Y, Baba T, Baba Y, et al. Dynamic contrast-enhanced MR imaging of uterine cervical cancer: pharmacokinetic analysis with histopathologic correlation and its importance in predicting the outcome of radiation therapy. Radiology 2000;216:803-09

27. George ML, Dzik-Jurasz AS, Padhani AR, et al. Non-invasive methods of assessing angiogenesis and their value in predicting response to treatment in colorectal cancer. Br J Surg 2001;88:1628-36

28. Loncaster JA, Carrington BM, Sykes JR, et al. Prediction of radiotherapy outcome using dynamic contrast enhanced MRI of carcinoma of the cervix. Int $J$ Radiat Oncol Biol Phys 2002;54:759-67

29. van Laarhoven HW, Klomp DW, Rijpkema M, et al. Prediction of chemotherapeutic response of colorectal liver metastases with dynamic gadoliniumDTPA-enhanced MRI and localized 19F MRS pharmacokinetic studies of 5-fluorouracil. NMR Biomed 2007;20:128-40

30. Cao Y, Popovtzer A, Li D, et al. Early prediction of outcome in advanced headand-neck cancer based on tumor blood volume alterations during therapy: a prospective study. Int J Radiat Oncol Biol Phys 2008;72:1287-90

31. Zima A, Carlos R, Gandhi D, et al. Can pretreatment CT perfusion predict response of advanced squamous cell carcinoma of the upper aerodigestive tract treated with induction chemotherapy? AJNR Am J Neuroradiol 2007;28:328-34

32. Gandhi D, Chepeha DB, Miller T, et al. Correlation between initial and early follow-up CT perfusion parameters with endoscopic tumor response in patients with advanced squamous cell carcinomas of the oropharynx treated with organ-preservation therapy. AJNR Am J Neuroradiol 2006;27:101-06

33. Hermans R, Meijerink M, Van den Bogaert W, et al. Tumor perfusion rate determined noninvasively by dynamic computed tomography predicts outcome in head-and-neck cancer after radiotherapy. Int J Radiat Oncol Biol Phys 2003;57:1351-56

34. Evelhoch JL, He Z, Polin L, et al. Dynamic contrast enhanced MRI evaluation of the effects of ZD6126 on tumor vasculature. In: Proceedings of the International Society for Magnetic Resonance in Medicine, Glasgow, Scotland, April 2127, 2001;9:481

35. Zhao J, Salmon H, Sarntinoranont M. Effect of heterogeneous vasculature on interstitial transport within a solid tumor. Microvasc Res 2007;73:224-36. Epub 2006 Dec 24

36. Pellerin M, Yankeelov TE, Lepage M. Incorporating contrast agent diffusion into the analysis of DCE-MRI data. Magn Reson Med 2007;58:1124-34

37. Zhou R, Pickup S, Yankeelov TE, et al. Simultaneous measurement of arterial input function and tumor pharmacokinetics in mice by dynamic contrast enhanced imaging: effects of transcytolemmal water exchange. Magn Reson Med 2004;52:248-57 